\title{
A CLASS OF MULTIVALENT FUNCTIONS WITH NEGATIVE TAYLOR COEFFICIENTS
}

\section{Introduction}

Let $D$ denote the unit disc $\{z:|z|<1\}$. Let $A_{p}, p=1,2, \ldots$, be the class of functions $f$ analytic in $D$ and represented by the Taylor series

$$
f(z)=z^{p}+\sum_{k=1}^{\infty} a_{k} z^{k+p}, \quad z \in D .
$$

A function $f$ in $A_{p}$ is said to be $p$-valent starlike of order $\alpha, 0 \leq \alpha<1$, if

$$
\operatorname{Re}\left\{\frac{z f^{\prime}(z)}{f(z)}\right\}>p a, \quad z \in D
$$

and $f$ is said to be a $p$-valent convex function of order $\alpha$, if $\frac{z f^{\prime}(z)}{p}$ is $p$-valent starlike of order $\alpha[1]$.

Let $S^{\circ}[p, \alpha]$ denote the class of $p$-valent starlike functions $f$ of order $\alpha$, given by the Taylor series

$$
f(z)=z^{p}-\sum_{k=1}^{\infty}\left|a_{k}\right| z^{k+p}, \quad z \in D .
$$

Similarly, let $K[p, \alpha]$ denote the class of $p$-valent convex functions of order $\alpha$ that are represented by (1.2). Kapoor and Mishra [3] have proved that a

Key words and phrases: p-valent starlike functions of order $a$, order of starlikeness, quasi-Hadamard product, distortion theorem etc.

AMS(MOS) subject classifications (1980): 30C 45.

* The research of the first author was supported by UGC New Delhi Major Research Project Grant No. F, -8-2/92 (SR-I), D 9.6.93.

** The research was done while the second author visited Berhampur University from S.K.C.G. College Paralakhemundi under UGC New Delhi, Teacher Fellowship Scheme, Grant No. F. 9-22/92 (CD-4) D 25.10.1993. 
function $f$ given by $(1.2)$ is in $S^{\circ}[p, \alpha]$ if and only if

$$
\sum_{k=1}^{\infty}(k+p-p \alpha)\left|a_{k}\right| \leq p(1-\alpha)
$$

and $f$ is in $K[p, \alpha]$ if and only if

$$
\sum_{k=1}^{\infty}\left(\frac{k+p}{p}\right)(k+p-p \alpha)\left|a_{k}\right| \leq p(1-\alpha) .
$$

For any real number $\lambda$, let $S_{\lambda}[p, \alpha], 0 \leq \alpha<1$, denote the class of functions $f$ given by the Taylor series (1.2) and satisfying

$$
\sum_{k=1}^{\infty}\left(\frac{k+p}{p}\right)^{\lambda}(k+p-p \alpha)\left|a_{k}\right| \leq p(1-\alpha) .
$$

It follows that $S_{0}[p, \alpha]=S^{\circ}[p, \alpha], S_{1}[p, \alpha]=K[p, \alpha]$ and if $\lambda_{1}<\lambda_{2}$, then $S_{\lambda_{2}}[p, \alpha] \subsetneq S_{\lambda_{1}}[p, \alpha]$. Thus, for $\lambda \geq 0$, functions in $S_{\lambda}[p, \alpha]$ are $p$-valent starlike of order $\alpha$ and further if $\lambda \geq 1$, functions in $S_{\lambda}[p, \alpha]$ are $p$-valent convex of order $\alpha$. For $\lambda \geq 0, p=1$, this class has been studied by Kumar [4]. For $\lambda<0, S_{\lambda}[p, \alpha]$ contains some non $p$-valent functions also.

EXAMPLE. Consider the functions $f_{k}$ in $S_{\lambda}[p, \alpha]$ given by

$$
f_{k}(z)=z^{p}-\frac{p^{\lambda+1}(1-\alpha)}{(k+p-p \alpha)(k+p)^{\lambda}} z^{k+p}, \quad z \in D,
$$

where $0 \leq \alpha<1, k, p=1,2, \ldots$ and $\lambda<0$. We shall show that, for fixed $\alpha$ and $p$, there exists a positive integer $k_{0}$ such that for all $k>k_{0}$, the $p$ th derivative of each function $f_{k}$ vanishes at some point in $D$, so that $f_{k}$ is not $p$-valent for $k>k_{0}$.

In fact, $f_{k}^{(p)}(z)$ is equal to zero, if

$$
z^{k}=\frac{p-1}{k+p-1} \frac{p-2}{k+p-2} \cdots \frac{1}{k+1} \frac{p^{-\lambda}(k+p-p \alpha)}{(k+p)^{1-\lambda}(1-\alpha)} .
$$

Thus, $|z|<1$ is satisfied if

$$
\frac{p^{-\lambda}(k+p-p \alpha)}{(k+p)^{1-\lambda}(1-\alpha)}<1
$$

which is equivalent to

$$
\alpha<\frac{(k+p)^{1-\lambda}-p^{-\lambda}(k+p)}{(k+p)^{1-\lambda}-p^{1-\lambda}}=F(k) .
$$

Note that $F(k)$ is increasing for $k>(p /(-\lambda))$ and $\lim _{k \rightarrow \infty} F(k)=1$. Thus, for fixed $\alpha$ and $p$, we can choose a $k_{0}$ such that (1.7) is satisfied for all 
$k>k_{0}>(p /(-\lambda))$. The function $F(k)$ is increasing what can be seen from the following calculations. $F(k)<F(k+1)$ is equivalent to

$$
\left(1+\frac{1}{k+p}\right)^{1-\lambda}-\left(1+\frac{1}{k}\right)+\frac{p^{1-\lambda}}{k(k+p)^{1-\lambda}}>0 .
$$

Since

$$
\left(1+\frac{1}{k+p}\right)^{1-\lambda}>1+\frac{1-\lambda}{k+p}
$$

it follows that $(1.8)$ is true if $k>(p /(-\lambda))$.

In the present paper we study the class $S_{\lambda}[p, \alpha]$ for all nonnegative as well as for some negative values of $\lambda$. In particular, we obtain distortion theorems, a covering theorem, order of starlikeness and order of convexity for the family $S_{\lambda}[p, \alpha]$. These results extend those of Silverman in [5]. Our result on quasi-Hadamard product of several functions in $S_{\lambda}[p, \alpha]$ unifies and generalizes some recent results of Kumar [4].

\section{Distortion and covering theorems}

It follows from (1.5) that if $f$ given by (1.2) is in $S_{\lambda}[p, \alpha]$, then

$$
\left|a_{k}\right| \leq \frac{(1-\alpha) p^{\lambda+1}}{(k+p-p \alpha)(k+p)^{\lambda}}
$$

Equality holds in $(2.1)$, for each $k=1,2, \ldots$, only for the functions

$$
f_{k}(z)=z^{p}-\frac{(1-\alpha) p^{\lambda+1}}{(k+p-p \alpha)(k+p)^{\lambda}} z^{k+p}, \quad z \in D .
$$

The function

$$
f_{1}(z)=z^{p}-\frac{(1-\alpha) p^{\lambda+1}}{(1+p-p \alpha)(1+p)^{\lambda}} z^{p+1}, \quad z \in D,
$$

is of foremost importance in the discussion of sharpness for the results of this paper.

We obtain the following results.

THEOREM 1. Let $f$ be in $S_{\lambda}[p, \alpha]$. Then for $\lambda \geq-1$

$$
\begin{aligned}
& |f(z)| \leq r^{p}+\frac{(1-\alpha) p^{\lambda+1}}{(1+p-p \alpha)(1+p)^{\lambda}} r^{1+p}, \\
& |f(z)| \geq r^{p}-\frac{(1-\alpha) p^{\lambda+1}}{(1+p-p \alpha)(1+p)^{\lambda}} r^{1+p},
\end{aligned}
$$

where $r=|z|$. Equality holds in (2.4) at $z=-r$ and in (2.5) at $z=r$ only for the function $f_{1}$ defined by (2.3). 
Proof. For $\lambda \geq-1,\left(\frac{k+p}{p}\right)^{\lambda}(k+p-p \alpha)$ is an increasing sequence in $k$. So,

$$
\begin{aligned}
p(1-\alpha) & \geq \sum_{k=1}^{\infty}\left(\frac{k+p}{p}\right)^{\lambda}(k+p-p \alpha)\left|a_{k}\right| \\
& \geq\left(\frac{1+p}{p}\right)^{\lambda}(1+p-p \alpha) \sum_{k=1}^{\infty}\left|a_{k}\right| .
\end{aligned}
$$

Equivalently,

$$
\sum_{k=1}^{\infty}\left|a_{k}\right| \leq \frac{(1-\alpha) p^{\lambda+1}}{(1+p-p \alpha)(1+p)^{\lambda}}
$$

Using (2.6), we have

$$
\begin{aligned}
|f(z)| & \leq|z|^{p}+\sum_{k=1}^{\infty}\left|a_{k}\right||z|^{k+p} \leq|z|^{p}+|z|^{1+p} \sum_{k=1}^{\infty}\left|a_{k}\right| \\
& \leq|z|^{p}+|z|^{1+p}\left(\frac{p}{1+p}\right)^{\lambda} \frac{p(1-\alpha)}{(1+p-p \alpha)} .
\end{aligned}
$$

Hence we get (2.4). Similarly

$$
|f(z)| \geq|z|^{p}-\sum_{k=1}^{\infty}\left|a_{k}\right||z|^{k+p} \geq|z|^{p}-|z|^{1+p} \sum_{k=1}^{\infty}\left|a_{k}\right|
$$

and, by (2.6), we obtain (2.5).

It is clear that equality holds in (2.4) and in (2.5) only if it holds in (2.6). However, in view of (1.5), this is true only if

$$
\left|a_{1}\right|=\frac{(1-\alpha) p^{\lambda+1}}{(1+p-p \alpha)(1+p)^{\lambda}}
$$

Thus, as indicated at the beginning of this section, $f$ must be equal to $f_{1}$ defined by (2.3). The proof is complete.

Theorem 2. For $-1 \leq \lambda<0$, let

$$
\alpha_{0}=1-\frac{p^{-\lambda}}{(1+p)^{1-\lambda}-p^{1-\lambda}} .
$$

The disc $|z|<1$ is mapped by any function in $S_{\lambda}[p, \alpha], \lambda \geq-1$, onto a domain that contains the disc

(a) $|w|<1-\frac{(1-\alpha) p^{\lambda+1}}{(1+p-p \alpha)(1+p)^{\lambda}} \equiv r_{1}(p, \alpha, \lambda)$, if $\lambda \geq 0$ and $0 \leq \alpha<1$ or $-1 \leq \lambda<0$ and $\alpha_{0}<\alpha<1$,

(b) $|w|<\frac{1}{p+1}\left[\frac{1+p-p \alpha}{(1+p)(1-\alpha)}\left(\frac{1+p}{p}\right)^{\lambda}\right]^{p} \equiv r_{2}(p, \alpha, \lambda)$, if $-1 \leq \lambda<0$ and $0 \leq \alpha<\alpha_{0}$. 
P r o of. First, note that, if $\lambda<0$, then

$$
0<\frac{p^{-\lambda}}{(1+p)^{1-\lambda}-p^{1-\lambda}}<1
$$

Therefore, $0<\alpha_{0}<1$. In view of Theorem $1(2.5)$, the range of $f$ contains the disc $|w|<\max (g(r)), 0<r<1$, where

$$
g(r)=r^{p}-\frac{p(1-\alpha)}{(1+p-p \alpha)}\left(\frac{p}{p+1}\right)^{\lambda} r^{p+1} .
$$

Differentiation with respect to $r$ gives

$$
g^{\prime}(r)=p r^{p-1}\left[1-\frac{(1+p)(1-\alpha)}{(1+p-p \alpha)}\left(\frac{p}{p+1}\right)^{\lambda} r\right]
$$

Note that $[(1+p)(1-\alpha) /(1+p-p \alpha)]<1$, if $\alpha>0$, and $(p /(1+p))^{\lambda} \leq 1$, if $\lambda \geq 0$. Therefore, $g$ is increasing in $r, 0<r<1$, if $0<\alpha<1$ and $\lambda \geq 0$, so $\max (g(r))=g(1)$. This gives the first part of $(\mathrm{a})$.

Next, if $-1 \leq \lambda<0$, then the condition

$$
\frac{(1+p)(1-\alpha)}{(1+p-p \alpha)}\left(\frac{p}{1+p}\right)^{\lambda} \leq 1
$$

is equivalent to $\alpha_{0} \leq \alpha$. Therefore, if $-1 \leq \lambda<0$ and $\alpha_{0} \leq \alpha<1$, then $g$ is increasing in $r, 0<r<1$. Thus $\max (g(r))=g(1)$. This gives the second part of (a).

On the other hand, if $-1 \leq \lambda<0$ and $0 \leq \alpha<\alpha_{0}$, then $g^{\prime}\left(r_{0}\right)=0$, where

$$
r_{0}=\left(\frac{p+1}{p}\right)^{\lambda} \frac{(1+p-p \alpha)}{(1+p)(1-\alpha)}<1
$$

and

$$
g^{\prime \prime}\left(r_{0}\right)=-p\left[\frac{(1+p-p \alpha)}{(1+p)(1-\alpha)}\left(\frac{1+p}{p}\right)^{\lambda}\right]^{p-2}<0 .
$$

Therefore,

$$
\max g(r)=g\left(r_{0}\right)=\frac{1}{(p+1)}\left[\frac{(1+p-p \alpha)}{(1+p)(1-\alpha)}\left(\frac{p+1}{p}\right)^{\lambda}\right]^{p}
$$

This gives (b). The proof is complete.

Remark 1 . By choosing $p=1, \lambda=0$ and $p=1, \lambda=1$ in Theorem 2 we get

$$
r_{1}(1, \alpha, 0)=\frac{1}{2-\alpha} \quad \text { and } \quad r_{1}(1, \alpha, 1)=\frac{3-\alpha}{4-2 \alpha}
$$


a result of Silverman [5, Theorem 5]. Further, for fixed $p$ and $\lambda,-1 \leq \lambda \leq 0$, a routine calculation shows that

$$
\frac{1}{(p+1)}=r_{1}\left(p, \alpha_{0}, \lambda\right)=\lim _{\alpha \rightarrow \alpha_{0}} r_{2}(p, \alpha, \lambda) \text {. }
$$

Theorem 3. For $f \in S_{\lambda}[p, \alpha], 0 \leq \alpha<1, \lambda \geq 0$, we have

$$
\begin{aligned}
& \left|f^{\prime}(z)\right| \leq p r^{p-1}\left(1+\frac{p^{\lambda}(1-\alpha)}{(1+p)^{\lambda-1}(1+p-p \alpha)} r\right), \\
& \left|f^{\prime}(z)\right| \geq p r^{p-1}\left(1-\frac{p^{\lambda}(1-\alpha)}{(1+p)^{\lambda-1}(1+p-p \alpha)} r\right),
\end{aligned}
$$

with $r=|z|$. Equality holds in (2.8) at $z=-r$ and in (2.9) at $z=r$ only for the function $f_{1}$ defined by (2.3).

Pr o of. Since

$$
(1+p-p \alpha) \sum_{k=1}^{\infty}\left(\frac{k+p}{p}\right)^{\lambda}\left|a_{k}\right| \leq \sum_{k=1}^{\infty}\left(\frac{k+p}{p}\right)^{\lambda}(k+p-p \alpha)\left|a_{k}\right| \leq p(1-\alpha)
$$

we have

$$
\sum_{k=1}^{\infty}\left(\frac{k+p}{p}\right)^{\lambda}\left|a_{k}\right| \leq \frac{p(1-\alpha)}{(1+p-p \alpha)}
$$

Using (1.5) and (2.10), we get

$$
\begin{gathered}
\left(\frac{1+p}{p}\right)^{\lambda} \sum_{k=1}^{\infty}(k+p)\left|a_{k}\right| \leq \sum_{k=1}^{\infty}\left(\frac{k+p}{p}\right)^{\lambda}(k+p)\left|a_{k}\right| \\
\quad=\sum_{k=1}^{\infty}\left(\frac{k+p}{p}\right)^{\lambda}(k+p-p \alpha)\left|a_{k}\right|+\sum_{k=1}^{\infty}\left(\frac{k+p}{p}\right)^{\lambda} p \alpha\left|a_{k}\right| \\
\leq p(1-\alpha)+p \alpha \frac{p(1-\alpha)}{(1+p-p \alpha)}=\frac{p(1+p)(1-\alpha)}{(1+p-p \alpha)}
\end{gathered}
$$

So,

$$
\sum_{k=1}^{\infty}(k+p)\left|a_{k}\right| \leq \frac{p^{\lambda+1}}{(1+p)^{\lambda-1}} \frac{(1-\alpha)}{(1+p-p \alpha)} .
$$

Using (2.11), we get for $|z|=r$

$$
\begin{aligned}
\mid f^{\prime}(z) & \leq p|z|^{p-1}+\sum_{k=1}^{\infty}(k+p)\left|a_{k}\right||z|^{k+p-1} \\
& \leq p r^{p-1}+r^{p} \sum_{k=1}^{\infty}(k+p)\left|a_{k}\right|
\end{aligned}
$$




$$
\begin{aligned}
& \leq p r^{p-1}+r^{p} \frac{p^{\lambda+1}}{(1+p)^{\lambda-1}} \frac{(1-\alpha)}{(1+p-p \alpha)} \\
& =p r^{p-1}\left(1+\frac{(1-\alpha) p^{\lambda}}{(1+p)^{\lambda-1}(1+p-p \alpha)} r\right) .
\end{aligned}
$$

This gives (2.8). Similarly,

$$
\begin{aligned}
\left|f^{\prime}(z)\right| & \geq p|z|^{p-1}-\sum_{k=1}^{\infty}(k+p)\left|a_{k}\right||z|^{k+p-1} \\
& \geq p r^{p-1}\left(1-\frac{(1-\alpha) p^{\lambda}}{(1+p-p \alpha)(1+p)^{\lambda-1}} r\right)
\end{aligned}
$$

This gives (2.9).

We note that equality in (2.10), hence in (2.11), holds only if (2.7) is true. So equality holds in (2.8) at $z=-r$ and in (2.9) at $z=r$ only for the function $f_{1}$ defined by (2.3). This completes the proof.

\section{Quasi-Hadamard product}

Let $f$ and $g$ be two functions analytic in $D$ and be represented by the Taylor series

$$
\begin{aligned}
& f(z)=a_{0} z^{p}-\sum_{k=1}^{\infty} a_{k} z^{k+p}, \quad a_{0}>0, a_{k} \geq 0, k=1,2, \ldots, \\
& g(z)=b_{0} z^{p}-\sum_{k=1}^{\infty} b_{k} z^{k+p}, \quad b_{0}>0, b_{k} \geq 0, k=1,2, \ldots
\end{aligned}
$$

The quasi-Hadamard product of $f$ and $g$ is defined to be the analytic function $f \diamond g$ given by. the Taylor series

$$
(f \circ g)(z)=a_{0} b_{0} z^{p}-\sum_{k=1}^{\infty} a_{k} b_{k} z^{k+p} .
$$

Note that the usual Hadamard product would give the Taylor series

$$
a_{0} b_{0} z^{p}+\sum_{k=1}^{\infty} a_{k} b_{k} z^{k+p}
$$

For $p=1$, the above definition of quasi-Hadamard product is due to $\mathrm{Ku}$ mar [4]. Quasi-Hadamard product of several functions is defined similarly. We next prove a theorem that generalizes and unifies some recent results of Kumar [4, Theorem A, B, C]. 
TheOrem 4. For $i=1,2, \ldots, m$ let $g_{i}$, given by

$$
g_{i}(z)=z^{p}-\sum_{k=1}^{\infty} a_{k, i} z^{k+p}, \quad a_{k, i} \geq 0, k=1,2, \ldots,
$$

be in $S_{\lambda_{i}}\left[p, \alpha_{i}\right], 0 \leq \alpha_{i}<1$ and $-\infty<\lambda_{i}<\infty$. Then, the function $g=$ $=g_{1} \diamond g_{2} \diamond \ldots \diamond g_{m}$ is in $S_{\mu}\left[p, \alpha^{\diamond}\right]$, where $\mu=\sum_{i=1}^{m} \lambda_{i}+(m-1)$ and $\alpha^{\circ}=$ $=\max \left\{\alpha_{1}, \alpha_{2}, \ldots, \alpha_{m}\right\}$.

Proof. It is sufficient to show that

$$
\sum_{k=1}^{\infty}\left[\left(\frac{k+p}{p}\right)^{\mu}\left(k+p-p \alpha^{\diamond}\right) \prod_{i=1}^{m} a_{k, i}\right] \leq p\left(1-\alpha^{\diamond}\right) .
$$

Without loss of generality we assume that $\alpha_{m}=\alpha^{\circ}$. Since $g_{i} \in S_{\lambda_{i}}\left[p, \alpha_{i}\right]$, we have

$$
\sum_{k=1}^{\infty}\left(\frac{k+p}{p}\right)^{\lambda_{i}}\left(k+p-p \alpha_{i}\right) a_{k, i} \leq p\left(1-\alpha_{i}\right)
$$

for every $i=1,2, \ldots, m$.

Therefore,

$$
a_{k, i} \leq \frac{p\left(1-\alpha_{i}\right)}{k+p-p \alpha_{i}}\left(\frac{p}{k+p}\right)^{\lambda_{i}} \leq\left(\frac{p}{k+p}\right)^{\lambda_{i}+1} .
$$

Using (3.2) for $i=1,2, \ldots, m-1$ and (3.1) for $i=m$, we obtain,

$$
\begin{aligned}
& \sum_{k=1}^{\infty} {\left[\left(\frac{k+p}{p}\right)^{\mu}\left(k+p-p \alpha^{\diamond}\right)\left(\prod_{i=1}^{m} a_{k, i}\right)\right] } \\
& \quad \leq \sum_{k=1}^{\infty}\left[\left(\frac{k+p}{p}\right)^{\mu}\left(k+p-p \alpha^{\diamond}\right)\left(\frac{p}{k+p}\right)^{\lambda_{1}+\ldots+\lambda_{m-1}+m-1} a_{k, m}\right] \\
& \quad=\sum_{k=1}^{\infty}\left[\left(\frac{k+p}{p}\right)^{\lambda_{m}}\left(k+p-p \alpha_{m}\right) a_{k, m}\right] \leq p\left(1-\alpha_{m}\right)=p\left(1-\alpha^{\diamond}\right) .
\end{aligned}
$$

Hence, $g=g_{1} \diamond g_{2} \diamond \ldots \diamond g_{m} \in S_{\mu}\left[p, \alpha^{\diamond}\right]$. The proof is complete.

Remark 2. Theorem 4 is new even in the case of $p=1$ and provides several interesting consequences. If we take $\lambda_{i}=0$ and $\lambda_{i}=1$ for $i=$ $=1,2, \ldots, m$ in Theorem 4 with $p=1$, we get Theorem $\mathrm{A}$ and Theorem $\mathrm{B}$ of [4], respectively. Similarly, the choice of $\lambda_{i}=0$ for $i=1,2, \ldots, q$ and $\lambda_{i}=1$ for $i=q+1, q+2, \ldots, m$ in Theorem 4 with $p=1$, gives Theorem $\mathrm{C}$ of $[4]$.

A general problem in the theory of univalent functions concerns the study of those transformations which carry one or several univalent as well 
as nonunivalent functions into the class of univalent functions. A useful consequence of Theorem 4 , related to the above problem is that quasiHadamard product of suitable number of nonunivalent functions can produce a univalent function. For example, in Theorem 4 with $p=1$, if we choose $-1+\frac{1}{m} \leq \lambda_{i} \leq 0, i=1,2, \ldots, m$, then the quasi-Hadamard product $f_{1} \diamond f_{2} \diamond \ldots \diamond f_{m}$ of functions $f_{i}$ in $S_{\lambda_{i}}[1, \alpha]$ is a starlike univalent function. Similarly, if $m \geq 2, i=1,2, \ldots, m$, and $\frac{2}{m}-1 \leq \lambda_{i} \leq 0$, then $f_{1} \diamond f_{2} \diamond \ldots \diamond f_{m}$, for $f_{i}$ in $S_{\lambda_{i}}[1, \alpha]$, is a convex univalent function. A yet simpler example gives that the quasi-Hadamard product of an univalent convex function given by (1.2) and a function in $S_{-1}[1, \alpha]$ is a convex univalent function. Similar remarks for $p$-valent cases also follow from Theorem 4 .

4. Order of starlikeness and order of convexity for $S_{\lambda}[p, \alpha]$

Silverman [4] has shown that the order of starlikeness of the family of univalent convex functions of order $\alpha$ is equal to $\frac{2}{3-\alpha}$. Kapoor and Mishra [3] have shown that, if $f$ given by (1.2) is in $K[p, \alpha]$, then $f$ is in $S^{\diamond}[p, \beta]$, where

$$
\beta \equiv \beta(\alpha)=\frac{p+1}{2 p+1-p \alpha} .
$$

Theorem 5. Suppose $-\infty<t<s<\infty, s-t \geq 1, p=1,2, \ldots$, and $1-\frac{1}{p^{2}} \leq \alpha<1$. If $f \in S_{s}[p, \alpha]$, then $f \in S_{t}[p, \beta]$, where

$$
\beta=\frac{\left(\frac{1+p}{p}\right)^{m}(1+p-p \alpha)-(1+p)(1-\alpha)}{\left(\frac{1+p}{p}\right)^{m}(1+p-p \alpha)-p(1-\alpha)}, \quad m=s-t
$$

The result is sharp with

$$
f(z)=z^{p}-\left(\frac{p}{1+p}\right)^{s} \frac{p(1-\alpha)}{(1+p-p \alpha)} z^{p+1}
$$

being extremal.

P roof. In view of (1.5), it suffices to show that

$$
\frac{\left(\frac{k+p}{p}\right)^{t}(k+p-p \beta)}{1-\beta} \leq \frac{\left(\frac{k+p}{p}\right)^{s}(k+p-p \alpha)}{1-\alpha}
$$

for all $k=1,2, \ldots$. This is equivalent to show that

$$
\beta \leq \frac{\left(\frac{k+p}{p}\right)^{s}(k+p-p \alpha)-\left(\frac{k+p}{p}\right)^{t}(k+p)(1-\alpha)}{\left(\frac{k+p}{p}\right)^{s}(k+p-p \alpha)-p\left(\frac{k+p}{p}\right)^{t}(1-\alpha)} .
$$

Equivalently

$$
\beta \leq \frac{\left(\frac{k+p}{p}\right)^{m}(k+p-p \alpha)-(k+p)(1-\alpha)}{\left(\frac{k+p}{p}\right)^{m}(k+p-p \alpha)-p(1-\alpha)}=F(k), \quad k=1,2, \ldots
$$


Since $F(k)$ is an increasing sequence of $k$ for $s-t \geq 1$ and $1-\frac{1}{p^{2}} \leq \alpha<1$, the above is true, if we take $\beta=F(1)$. In order to show that $F$ is an increasing sequence of $k$, extend $F$ as a function of the real variable $x$. We get that $F^{\prime}(x)$ is equal to the expression given below

$$
\begin{aligned}
& \left\{( x + p ) ^ { s + t - 1 } p ^ { s + t } \left[(s-t)(x+p)^{2}-p(\alpha(s-t-1)+s-t+1)(x+p)\right.\right. \\
& \left.\left.\quad+\alpha p^{2}(s-t)\right]+(1-\alpha) p^{2 s+1}(x+p)^{2 t}\right\}(1-\alpha)\left[p^{t}(x+p)^{s+1}\right. \\
& \left.-\alpha p^{t+1}(x+p)^{s}-p^{s+1}(x+p)^{t}+\alpha p^{s+1}(x+p)^{t}\right]^{-2}
\end{aligned}
$$

Put $g(r)=(s-t) r^{2}-p(\alpha(s-t-1)+s-t+1) r+\alpha p^{2}(s-t)$. Then

$$
\begin{aligned}
g(0) & =\alpha p^{2}(s-t)>0, \\
g(p) & =-p^{2}(1-\alpha)<0, \\
g(1+p) & =(s-t)(1+p-p \alpha)-p(1+p)(1-\alpha) .
\end{aligned}
$$

Since $s-t \geq 1$ and $\alpha \geq 1-\frac{1}{p^{2}}$, we get $g(1+p)>0$, so both the roots of $g(r)$ lie in the interval $(0,1+p)$ and it follows that $g(r)>0$ for $r \geq 1+p$. in $k$.

This gives that $F^{\prime}(x)>0$ for $x \geq 1$ and $F(k)$ in an increasing sequence

A calculation gives that the $(p+1)$ coefficient of the function $f$ in $S_{s}[p, \alpha]$ given by (4.2) satisfies

$$
\left(\frac{1+p}{p}\right)^{t} \frac{(1+p-p \beta)}{p(1-\beta)}\left(\frac{p}{1+p}\right)^{s} \frac{p(1-\alpha)}{(1+p-p \alpha)}=1
$$

where $\beta$ is given by (4.1). Therefore, in view of (1.5), $f$ is in $S_{t}[p, \beta]$. Hence the value of $\beta$ given by (4.1) is the largest.

Remark 3. Theorem 5 has many interesting consequences in the case of $p=1$ also.

(a) Taking $t=0$ and $s \geq 1$, we get that the order of (univalent) starlikeness of any function in $S_{s}[1, \alpha] \equiv S_{s}[\alpha], 0 \leq \alpha<1$, is equal to

$$
\frac{2^{s}(2-\alpha)-2(1-\alpha)}{2^{s}(2-\alpha)-(1-\alpha)}
$$

The choice $s=1$ in (4.4) gives the result of Silverman [5, Theorem 7].

(b) Taking $t=1$ and $s \geq 2$, we get that the order of (univalent) convexity for any function in $S_{s}[1, \alpha]=S_{s}[\alpha], 0 \leq \alpha<1$, is equal to

$$
\frac{2^{s}(2-\alpha)-4(1-\alpha)}{2^{s}(2-\alpha)-2(1-\alpha)}
$$


(c) Taking $t=0$ and $s \geq 1$, we get that the order of $p$-valent starlikeness of any function in $S_{s}[p, \alpha], 1-\frac{1}{p^{2}} \leq \alpha<1$, is equal to

$$
\frac{\left(\frac{1+p}{p}\right)^{s}(1+p-p \alpha)-(1+p)(1-\alpha)}{\left(\frac{1+p}{p}\right)^{s}(1+p-p \alpha)-p(1-\alpha)} .
$$

The choice $s=1$ in (4.6) gives the result of Kapoor and Mishra [3, Theorem 4(i)].

(d) Taking $t=1$ and $s \geq 2$, we get that the order of $p$-valent convexity for any function in $S_{s}[p, \alpha], 1-\frac{1}{p^{2}} \leq \alpha<1$, is equal to

$$
\frac{\left(\frac{1+p}{p}\right)^{s}(1+p-p \alpha)-\left(\frac{1+p}{p}\right)(1+p)(1-\alpha)}{\left(\frac{1+p}{p}\right)^{s}(1+p-p \alpha)-\left(\frac{1+p}{p}\right) p(1-\alpha)} \text {. }
$$

In our next theorem we determine the sharp value of a constant $\gamma \equiv \gamma(\alpha)$ such that

$$
\operatorname{Re}\left\{\frac{f(z)}{z^{p}}\right\}>p \gamma
$$

for $f$ in $S_{\lambda}[p, \alpha], \lambda \geq-1$ and $z \in D$. We note that in general, if we do not assume the coefficients to be negative, then there does not exist any positive constant $\gamma$ such that

$$
\operatorname{Re}\left\{\frac{f(z)}{z^{p}}\right\}>p \gamma
$$

for $p$-valent convex functions of order $\alpha$ [2].

TheOREM 6. Let $f$, given by the Taylor series (1.2), be in $S_{\lambda}[p, \alpha]$, for $\lambda \geq-1$. Then

$$
\operatorname{Re}\left\{\frac{f(z)}{z^{p}}\right\}>p \gamma
$$

where

$$
\gamma \equiv \gamma(\alpha)=1-\left(\frac{p}{1+p}\right)^{\lambda} \frac{(1-\alpha)}{(1+p-p \alpha)} .
$$

The above result is sharp, the function $f_{1}$ defined by (2.3) being extremal.

Proof. Kapoor and Mishra [3] have shown that a function $f$ given by (1.2) satisfies (4.8) if and only if

$$
\sum_{k=1}^{\infty} \frac{\left|a_{k}\right|}{p(1-\gamma)} \leq 1
$$

Since $f$ is in $S_{\lambda}[p, \alpha]$, we have 


$$
\sum_{k=1}^{\infty}\left(\frac{k+p}{p}\right)^{\lambda} \frac{(k+p-p \alpha)}{p(1-\alpha)}\left|a_{k}\right| \leq 1
$$

Thus, it is sufficient to show that

$$
\frac{1}{p(1-\gamma)} \leq\left(\frac{k+p}{p}\right)^{\lambda} \frac{(k+p-p \alpha)}{p(1-\alpha)}, \quad \text { for all } k=1,2, \ldots
$$

This is equivalent to

$$
\gamma \leq 1-\left(\frac{p}{k+p}\right)^{\lambda} \frac{(1-\alpha)}{(k+p-p \alpha)}=\phi(k), \quad k=1,2, \ldots
$$

A brief calculation shows that $\phi(k)$ is an increasing sequence in $k$, if $\lambda \geq-1$. Thus we get (4.9) by taking $\gamma=\phi(1)$. We note that

$$
\frac{(1-\alpha) p^{\lambda+1}}{(1+p-p \alpha)(1+p)^{\lambda}} \frac{1}{p(1-\gamma)}=1
$$

where $\gamma$ is given by (4.9). Therefore, in view of (4.10), the value of $\gamma$ defined by $(4.9)$ is the largest. This completes the proof.

Acknowledgement. The authors thank the referee for his valuable suggestions which improved the final form of the manuscript.

\section{References}

[1] A. W. Goodman, On the Schwarz-Christoffel transformation and p-valent functions, Trans. Amer. Math. Soc. 68 (1950), 204-223.

[2] D. J. Hallenbeck, A. E. Livingston, Applications of extreme point theory to classes of multivalent functions, Trans. Amer. Math. Soc. 221 (1976), 339-359.

[3] G. P. Kapoor, A. K. Mishra, $a^{\diamond}-$ families of analytic functions, Internat. J. Math. and Math. Sci. 7 (1984), 435-442.

[4] V. Ku mar, Quasi-Hadamard product of certain univalent functions, J. Math. Anal. Appl. 126 (1987), 70-77.

[5] H. Silverm a n, Univalent functions with negative coefficients, Proc. Amer. Math. Soc. 51 (1975), 109-116.
A. K. Mishra
P. G. DEPARTMENT OF MATHEMATICS
BERHAMPUR UNIVERSITY
P.O. BHANJA BIHAR, (GANJAM DIST.)
ORISSA, INDIA - 760 007;
M. Choudhury
DEPARTMENT OF MATHEMATICS
GOVERNMENT COLLEGE PHULBANI
P.O., DIST. PHULBANI
ORISSA, INDIA

Received August 25, 1992; revised version December 6., 1999. 